\title{
等离子活化烧结制备石墨烯/差基磷灰石复相生物陶瓷
}

\author{
张彪, 杨长安, 施佩 \\ (陕西科技大学 材料科学与工程学院, 西安 710021)
}

摘 要: 以羟基磷灰石(Hydroxyapatite, HAp)为基体, 石墨烯(Graphene, rGO)作为增强相, 利用等离子活化烧结制备 了石墨烯/差基磷灰石 $(\mathrm{rGO} / \mathrm{HAp})$ 复相生物陶瓷。系统研究了 $\mathrm{rGO}$ 添加量对 $\mathrm{HAp}$ 陶瓷基体物相结构、生物活性及断 裂韧性的影响。结果表明, rGO 的加入有利于提高 HAp 陶瓷的生物活性。同时, 复相生物陶瓷的硬度与断裂韧性随 $\mathrm{rGO}$ 添加量的增加均表现出先升高, 后显著降低的变化趋势。当 $\mathrm{rGO}$ 添加量为 $2 \mathrm{wt} \%$ 时, 样品的硬度与断裂韧性分 别达到 $6.97 \mathrm{GPa}$ 和 $0.84 \mathrm{MPa} \cdot \mathrm{m}^{1 / 2}$, 较纯相 HAp 陶瓷提高了 $11.5 \%$ 和 $37.3 \%$ 。研究表明 $\mathrm{rGO}$ 的拔出效应是导致复相 陶瓷力学性能提高的主要原因。

关＼cjkstart键 词: 羟基磷灰石; 石墨烯; 等离子活化烧结; 生物活性; 断裂韧性

中图分类号: TB321 文献标识码: A

\section{Synthesis of Graphene/Hydroxyapatite Composite Bioceramics via Plasma Activated Sintering}

\author{
ZHANG Biao, YANG Chang-An, SHI Pei \\ (School of Materials Science \& Engineering, Shaanxi University of Science \& Technology, Xi'an 710021, China)
}

\begin{abstract}
Graphene/hydroxyapatite ( $\mathrm{rGO} / \mathrm{HAp}$ ) composite bioceramics were fabricated by plasma activated sintering using hydroxyapatite ( $\mathrm{HAp}$ ) as matrix and graphene (rGO) as reinforced phase. The effects of rGO addition on phase structure, bioactivity and fracture toughness of HAp ceramics matrix were systematically investigated. The results indicate that the incorporation of $\mathrm{rGO}$ is beneficial to promoting the bioactivity of HAp ceramics. Meanwhile, hardness and fracture toughness of composite bioceramics initially increase and then decrease significantly with rGO concentration increasing. The hardness and fracture toughness of the specimen with rGO loading of $2 \mathrm{wt} \%$ reach $6.97 \mathrm{GPa}$ and $0.84 \mathrm{MPa} \cdot \mathrm{m}^{1 / 2}$, which display $\sim 11.5 \%$ and $\sim 37.3 \%$ improvements as compared to pure HAp ceramics, respectively. It is demonstrated that the pull-out effect of rGO is the major reason for enhancing the mechanical properties of composite ceramics.
\end{abstract}

Key words: hydroxapatite; graphene; plasma activated sintering; bioactivity; fracture toughness

羟基磷灰石(Hydroxyapatite, HAp)是一类典型 的生物活性陶瓷，与人体骨骼和牙齿的化学成分和 结构相似，具有良好的生物活性、生物相容性和骨
传导性 ${ }^{[1]}$ 。但是在临床应用过程中, HAp 也显示了机 械强度低、抗瘦劳性差等缺点, 其断裂韧性 $\left(K_{\mathrm{IC}}\right)$ 仅 为 $0.5 \sim 0.7 \mathrm{MPa} \cdot \mathrm{m}^{1 / 2}$, 严重制约了其在承重部位骨替

收稿日期: 2018-04-02; 收到修改稿日期：2018-04-26

基金项目：陕西省科技统筹创新工程项目(2017TSCXL-GY-07-05, 2015KTTSGY02-03); 陕西科技大学博士科研启动基金 (2017BJ-26); 陕西科技大学研究生创新基金项目

Shaanxi Science \& Technology Co-ordination \& Innovation Project (2017TSCXL-GY-07-05, 2015KTTSGY02-03); Doctoral Scientific Research Fund of Shaanxi University of Science \& Technology (2017BJ-26); Graduate Innovation Fund of Shaanxi University of Science and Technology

作者简介: 张 彪(1990-), 男, 博士研究生. E-mail: yunzhuangzhb@163.com

通讯作者: 杨长安, 讲师. E-mail: yangchangan@sust.edu.cn 
换中的应用 ${ }^{[2-3]}$ 。如何在不降低 HAp 优良生物性能 的前提下, 更大限度地改善其力学性能, 尚有待深 入研究。

石墨烯(Graphene, rGO)作为一种新型二维碳材 料, 具有优异的机械性能(杨氏模量 $100 \mathrm{GPa}$, 断裂 强度 $125 \mathrm{GPa})$ 和较大的比表面积 $\left(2630 \mathrm{~m}^{2} / \mathrm{g}\right)$, 广泛应 用于复合材料增强相 ${ }^{[4-5]}$ 。已有研究表明: rGO 在人 体环境中生物相容性良好, 无毒性 ${ }^{[6]}$, 可以显著调 节细胞的黏附、增殖及分化行为 ${ }^{[7]}$, 在骨再生与修 复 ${ }^{[8]}$ 、药物靶向输送 ${ }^{[9]}$ 、细胞成像 ${ }^{[10]}$ 等方面具有潜 $^{\circ}$ 在的应用。

等离子活化烧结(Plasma Activated Sintering, PAS) 是近年发展起来的先进烧结技术, 具有烧结温度 低、升温速率快、保温时间短等优点 ${ }^{[11]}$ 。快速烧结 的特点可以有效抑制晶粒长大, 提高材料致密度, 因此, 很多研究致力于 PAS 烧结制备力学性能优异 的 HAp 生物陶瓷 ${ }^{[12-14]}$ 。

为改善 HAp 陶瓷的本征脆性和致密度差等问 题, 本研究利用等离子活化烧结技术制备了石墨烯/ 差基磷灰石 $(\mathrm{rGO} / \mathrm{HAp})$ 复相生物陶瓷, 进而探讨了 $\mathrm{rGO}$ 对 HAp 陶瓷物相结构、生物活性及断裂韧性的 影响规律。

\section{1 实验方法}

\section{$1.1 \mathrm{rGO} / \mathrm{HAp}$ 生物陶瓷的制备}

以 $\mathrm{Ca}\left(\mathrm{NO}_{3}\right)_{2} \cdot 4 \mathrm{H}_{2} \mathrm{O}$ 和 $\left(\mathrm{NH}_{4}\right)_{2} \mathrm{HPO}_{4}$ 分别作为 $\mathrm{Ca}$ 源和 $\mathrm{P}$ 源, 氨水和冰醋酸作为 $\mathrm{pH}$ 调节剂, 通过室温 共沉淀法制备 HAp 粉末, 采用改进的 Hummers 法 ${ }^{[15]}$ 制备氧化石墨烯(Graphene Oxide, GO), 进而通过水 合肼还原得到 $\mathrm{rGO}$ 。按照 $\mathrm{rGO}$ 添加量为 $0 、 1 \mathrm{wt} \%$ 、 $2 \mathrm{wt} \%$ 和 $5 \mathrm{wt} \%$, 将其与 HAp 粉体混合, 湿法球磨 $2 \mathrm{~h}$, 转速 $400 \mathrm{r} / \mathrm{min}, 60^{\circ} \mathrm{C}$ 真空干燥后获得分散均匀的 $\mathrm{rGO} / \mathrm{HAp}$ 复合粉体。

利用等离子活化烧结炉(ED-PASIII, JAPAN)烧 结制备 $\mathrm{rGO} / \mathrm{HAp}$ 复相生物陶瓷。具体烧结工艺为: 施加压力 $50 \mathrm{MPa}$, 以 $100^{\circ} \mathrm{C} / \mathrm{min}$ 升温至 $950^{\circ} \mathrm{C}$, 保温 $5 \mathrm{~min}$, 自然冷却至室温。将样品研磨抛光, 以备表 征测试。

\section{2 样品表征与性能测试}

采用扫描电镜(HITACHI S-4800, SEM)观察样 品的断口形貌及类骨磷灰石层; 采用 $\mathrm{X}$ 射线衍射 仪(RIGAKU D/MAX 2200PC, XRD)对 rGO/HAp 复 相陶瓷进行物相分析; 采用激光共聚焦拉曼光谱仪 (INVIA, Raman)研究 rGO 添加对 HAp 结构稳定性的 影响; 采用 X 射线能谱仪(EDS)分析 rGO 在 HAp 基
体中的分散状态; 采用激光扫描共聚焦显微镜(KEYENCE VK-9700, LSCM) 观测生物活性试验后样品 表面沉积层分布及厚度。采用模拟体液(Simulated Body Fluid, SBF)体外浸泡法评价样品的生物活性 ${ }^{[16]}$; 采用压痕法测试样品的显微硬度和断裂韧性 ${ }^{[17]}$ 。

\section{2 结果与讨论}

\section{$2.1 \mathrm{rGO} / \mathrm{HAp}$ 复相生物陶瓷的形貌分析}

图 1 为 $\mathrm{rGO} / \mathrm{HAp}$ 复相生物陶瓷的光学照片及 SEM 照片。PAS 烧结后样品结构致密, 呈现半乳浊 状态。添加 $\mathrm{rGO}$ 后, 样品白度逐渐下降, 表明利用 PAS 工艺的快速烧结致密化优势, 可以有效避免 $\mathrm{rGO}$ 的氧化挥发与结构破坏 ${ }^{[18]}$, 使 $\mathrm{rGO}$ 很好的保留 在 HAp 基体中, 从而更大限度地发挥其优异性能。 从图 1(a)中断口 SEM 照片可以看出, PAS 烧结后, 纯相 HAp 陶瓷晶粒粗大 (约为 3 4 $\mu \mathrm{m}$ ), 且晶界处存 在大量气孔, 致密度受到严重影响。当添加 $1 \mathrm{wt} \%$ 的 $\mathrm{rGO}$ 时, HAp 晶粒尺寸减小至 $3 \mu \mathrm{m}$ 左右, 断裂面呈层 状近弧形面, 表现为典型的贝壳状断口形貌(图 1(b))。 当 $\mathrm{rGO}$ 加入量增大至 $2 \mathrm{wt} \%$ 时, 可以明显观察到晶 粒度大大减小, 仅有 $300 \mathrm{~nm}$ 左右, 说明 $\mathrm{rGO}$ 的存在 可以明显细化 $\mathrm{HAp}$ 的晶粒, 这是由于 $\mathrm{rGO}$ 在陶瓷基 体中倾向于分布在 HAp 晶界处, 可以有效抑制晶界 迁移 ${ }^{[19]}$ 。此外, 复相陶瓷断面致密无气孔, 晶粒逐 渐发育为等轴晶。从其局部放大照片可以看到, 在 $\mathrm{HAp}$ 晶界处凸起许多片状晶体, 这是 rGO 沿晶界处 拔出所致。至 $\mathrm{rGO}$ 加入量变为 $5 \mathrm{wt} \%$, 样品晶粒度 没有发生明显变化,表现出典型的“冰糖状” 断口特 征, 说明此时主要发生沿晶断裂, 样品脆性较大。另 外,在晶界处产生少许气孔, 这可能是由于rGO 含量 过高被少量氧化所导致。
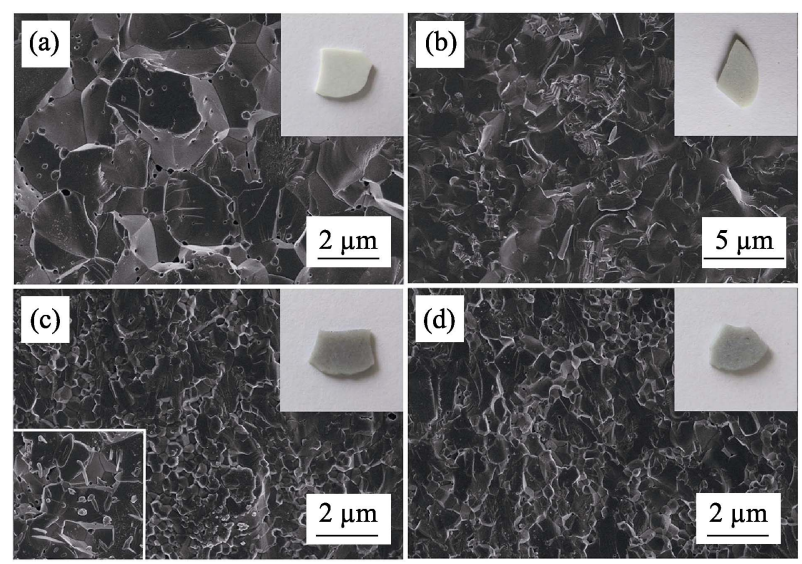

图 $1 \mathrm{rGO} / \mathrm{HAp}$ 复相生物陶瓷的光学照片及断口 SEM 照片 Fig. 1 Optical photos (insets) and SEM images of fracture surface of $\mathrm{rGO} / \mathrm{HAp}$ composite bioceramics

(a) Pure HAp; (b) $1 \mathrm{wt} \% \mathrm{rGO} / \mathrm{HAp}$; (c) $2 \mathrm{wt} \% \mathrm{rGO} / \mathrm{HAp}$; (d) $5 \mathrm{wt} \%$ $\mathrm{rGO} / \mathrm{HAp}$ 


\section{$2.2 \mathrm{rGO} / \mathrm{HAp}$ 复相生物陶瓷的物相及结构分析}

图 2 为 $\mathrm{rGO} / \mathrm{HAp}$ 复相生物陶瓷的 XRD 图谱。 可以明显观察到: $\mathrm{rGO}$ 添加量为 0 和 $1 \mathrm{wt} \%$ 的两个样 品中, 主晶相表现为 HAp 的特征, 且衍射峰相对强 度高, 结晶性能良好, 表明 PAS 在 $950^{\circ} \mathrm{C}$ 低温烧结 仍可达到高结晶高致密化的 HAp 陶瓷。同时, 样品 中出现少许 $\beta$-磷酸三钻 $(\beta$-TCP) 的衍射峰, 证明了此 时 HAp 发生了部分分解。而当 rGO 加入量为 $2 \mathrm{wt} \%$ 和 $5 \mathrm{wt} \%$ 时, 制备产物完全表现出 HAp 的特征峰, 并无 $\beta$-TCP 物相出现。已有文献表明 ${ }^{[20]}, \mathrm{HAp}$ 在高 温下会脱羟基, 分解产生 $\beta$ - TCP。由此可以推断: PAS 烧结前期的活化阶段, 脉冲电流产生的等离子 体使得 HAp 过热活化, 促使 HAp 脱羟基分解。而 $\mathrm{rGO}$ 纳米薄片可以起到将 HAp 晶粒隔离包裹的作 用，有效地抑制了 HAp 高温时的相分解，从而提高 其结构稳定性。

为了进一步验证 rGO 对 HAp 相分解的抑制作 用, 对所制备的样品进行 Raman 光谱表征, 如图 3 所示。由图可见, $\mathrm{rGO}$ 在 1350 和 $1580 \mathrm{~cm}^{-1}$ 处具有 2 个比较明显的特征峰, 分别对应 $\mathrm{rGO}$ 的 $\mathrm{D}$ 峰和 $\mathrm{G}$ 峰 ${ }^{[21]}$ 。在 $\mathrm{rGO} / \mathrm{HAp}(\mathrm{rGO}$ 添加量分别为 $2 \mathrm{wt} \%$ 和 $5 \mathrm{wt} \%$ ) 两个样品中, 均可观察到 $962.2 \mathrm{~cm}^{-1}$ 处强而尖锐的谱 峰, 这是磷酸盐中 $\mathrm{P}-\mathrm{O}$ 键的对称伸缩振动 $\mathrm{v}$ 引起的, 表明 $\mathrm{HAp}$ 符合标准化学计量比 $(\mathrm{Ca} / \mathrm{P}$ 原子比为 $1.67)^{[22]}$ 。但在 $\mathrm{rGO}$ 添加量为 0 和 $1 \mathrm{wt} \%$ 样品中, $\mathrm{P}-\mathrm{O}$ 键的对称伸缩振动峰向高波段移动, 分别出现在 $969.6 \mathrm{~cm}^{-1}$ 和 $971.1 \mathrm{~cm}^{-1}$, 说明此时合成的产物偏离 HAp 的标准化学计量比, 可能衍生为其他钙磷化合 物。同时, $950.3 \mathrm{~cm}^{-1}$ 处出现的谱峰代表 $\left[\mathrm{PO}_{4}\right]$ 的 $v 1$ 振动模式 ${ }^{[23]}$, 这是 $\beta$-TCP 区别于 HAp 所独有的。此 外, 从局部放大图可以发现, rGO 的特征峰依然存 在, 特别是在 $\mathrm{rGO}$ 添加量为 $5 \mathrm{wt} \%$ 时, 峰形较明显, 间接证明了 rGO 的存在可以抑制 HAp 的分解。

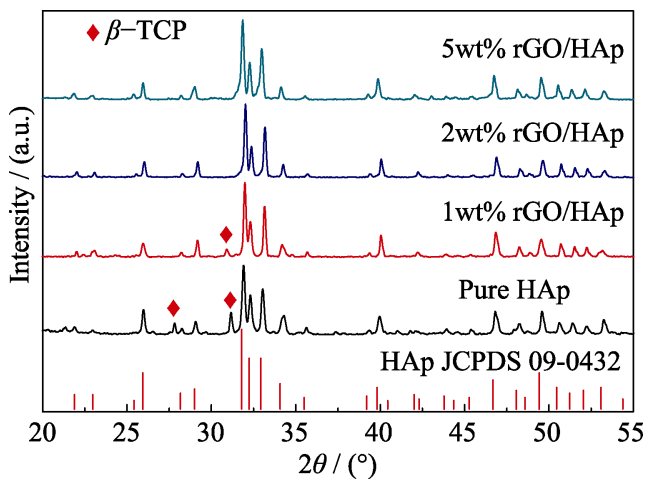

图 $2 \mathrm{rGO} / \mathrm{HAp}$ 复相生物陶瓷的 XRD 图谱

Fig. 2 XRD patterns of $\mathrm{rGO} / \mathrm{HAp}$ composite bioceramics

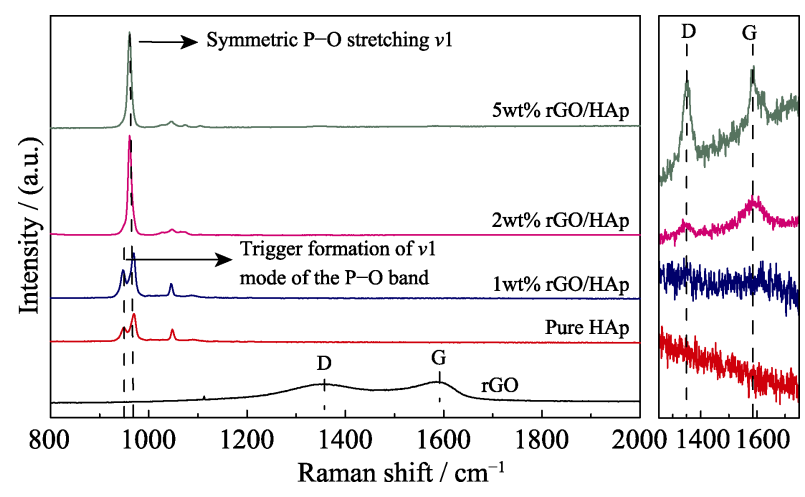

图 $3 \mathrm{rGO} / \mathrm{HAp}$ 复相生物陶瓷的 Raman 光谱图

Fig. 3 Raman spectra of rGO/HAp composite bioceramics

\section{$2.3 \mathrm{rGO} / \mathrm{HAp}$ 复相生物陶瓷的元素面分布分析}

图 4 表示 $2 \mathrm{wt} \% \mathrm{rGO} / \mathrm{HAp}$ 复相生物陶瓷断面 SEM 照片及 EDS 图谱。从图 4(a)中可以看出, $2 \mathrm{wt} \%$ $\mathrm{rGO} / \mathrm{HAp}$ 的组分晶粒紧密嵌合, 界面相互作用强, 致密度较高, 片状 rGO 从 $\mathrm{HAp}$ 基体中脱粘并拔出, 起到很好的增韧效果, 这与图 1(c)描述的基本一致。 为了研究 $\mathrm{rGO}$ 在 $\mathrm{HAp}$ 基体中的分散效果, 对样品进 行 EDS 分析。从图 4(b)可以看出, 基体主要由 C、 $\mathrm{O} 、 \mathrm{P} 、 \mathrm{Ca}$ 等元素组成, 其中 $\mathrm{Ca} / \mathrm{P}$ 原子比为 1.64 , 接 近 HAp 的理论比值, 说明 PAS 烧结后, 样品几乎没 有发生分解。 $\mathrm{C}$ 与 $\mathrm{O}$ 元素的存在证明了 $\mathrm{GO}$ 前驱体 还原不彻底, 或者 PAS 烧结过程中 rGO 发生了部分 氧化。此外, 从图 4(c) (e)中可以看出, 石墨烯在 HAp 基体中分散性良好, 这对提高复相陶瓷综合性 能是至关重要的。

\section{$2.4 \mathrm{rGO} / \mathrm{HAp}$ 复相生物陶瓷的生物活性评价}

将制备得到的样品浸泡于 SBF 溶液 $14 \mathrm{~d}$ 评价其 生物活性, 图 5 为 $\mathrm{rGO} / \mathrm{HAp}$ 复相生物陶瓷表面矿化 产物的 LSCM 照片。从图 5(a)中可以观察到, 纯相 HAp 表现出优异的生物活性, 这是 HAp 的组成及结 构所决定的。随着 rGO 添加量的增加, 复合材料的 生物活性表现出先降低再逐渐升高的趋势, 当 $\mathrm{rGO}$ 加入量为 $2 \mathrm{wt} \%$ 和 $5 \mathrm{wt} \%$ 的时候, 样品表面磷灰石层 的最大沉积厚度分别达到 19.8 和 $30.0 \mu \mathrm{m}$, 相比纯 相 HAp 致密陶瓷表现出更加优越的生物活性。证明 了 rGO 的添加并未对 HAp 的生物活性产生影响, 反 而会进一步改善 HAp 基体的生物活性。

进一步采用 SEM 表征矿化后样品的表面沉积 物, 如图 6 所示。可以看出, 矿化后, 复相陶瓷表面 出现大量均一的类骨磷灰石沉积产物, 从图 6(b)的 局部放大照片可以看出, 表面沉积物是由大量片状 晶体相互交织构成, 呈三维多孔网状结构, 与骨组 织工程支架极为相似，这种类骨磷灰石沉积层可以 诱导更多的成骨细胞在其表面增殖与分化 ${ }^{[24]}$, 表现 出较好的生物活性。 

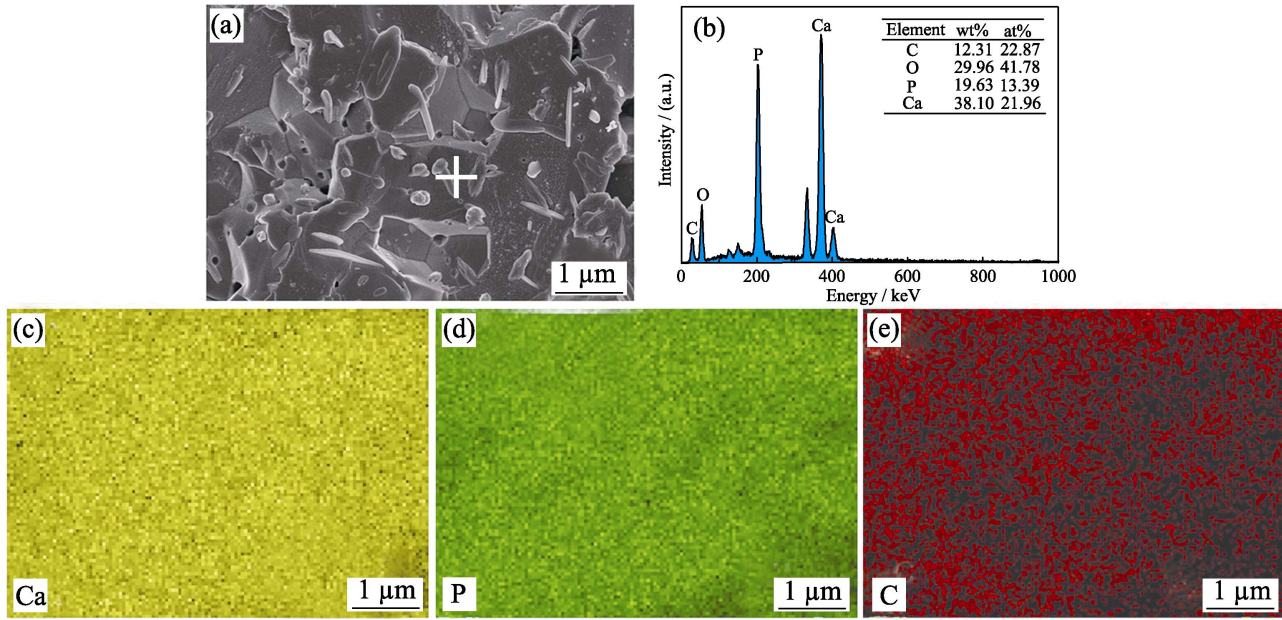

图 $42 \mathrm{wt} \% \mathrm{rGO} / \mathrm{HAp}$ 复相生物陶瓷的断口 SEM 照片及 EDS 图谱

Fig. 4 SEM images and EDS spectra of fracture surface of $2 \mathrm{wt} \% \mathrm{rGO} / \mathrm{HAp}$ composite bioceramics (a) SEM images; (b) EDS spectra; Elemental mappings of (c) Ca, (d) P, and (e) $\mathrm{C}$ elements
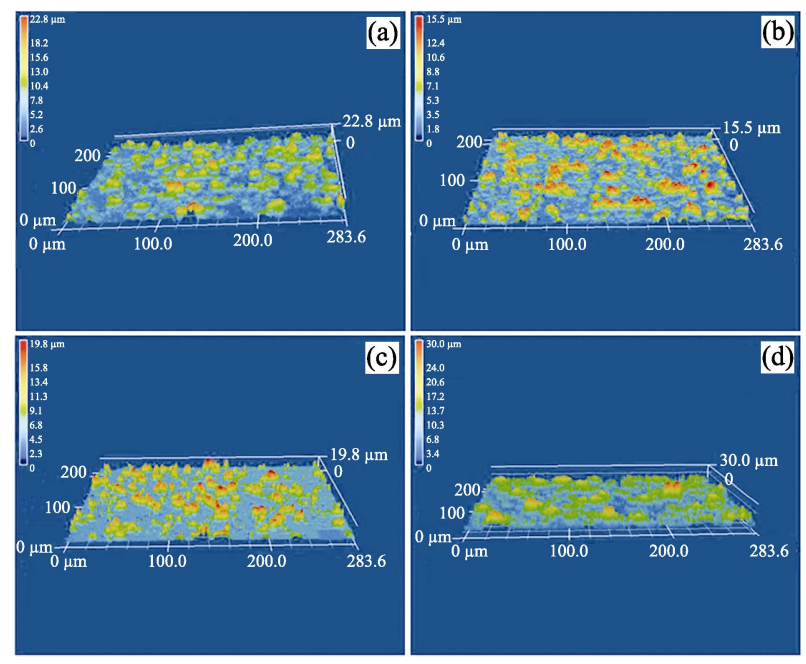

图 $5 \mathrm{rGO} / \mathrm{HAp}$ 复相生物陶瓷表面矿化产物的 LSCM 照片 Fig. 5 LSCM images of mineralization products on the surface of $\mathrm{rGO} / \mathrm{HAp}$ composite bioceramics

(a) Pure HAp; (b) $1 \mathrm{wt} \% \mathrm{rGO} / \mathrm{HAp}$; (c) $2 \mathrm{wt} \% \mathrm{rGO} / \mathrm{HAp}$; (d) $5 \mathrm{wt} \%$ $\mathrm{rGO} / \mathrm{HAp}$

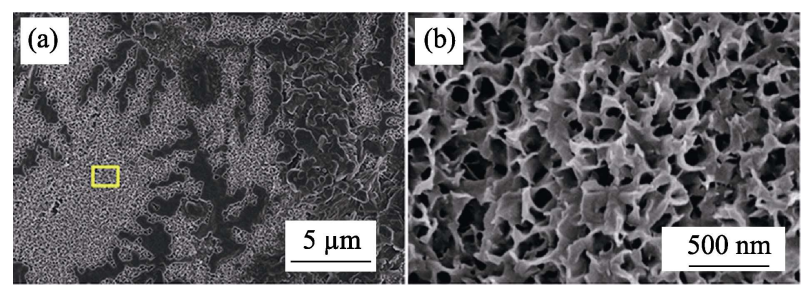

图 $62 \mathrm{wt} \% \mathrm{rGO} / \mathrm{HAp}$ 复相生物陶瓷表面沉积物的 SEM 照片

Fig. 6 SEM images of deposition on the surface of $2 \mathrm{wt} \%$ $\mathrm{rGO} / \mathrm{HAp}$ composite bioceramics

(a) Morphology of the surface sediment after mineralization; (b) Partial enlarged drawing

\section{$2.5 \mathrm{rGO} / \mathrm{HAp}$ 复相生物陶瓷的力学性能分析}

图 7 为 $\mathrm{rGO} / \mathrm{HAp}$ 复相生物陶瓷维氏硬度与断裂 㓞性的变化曲线。观察压痕照片首先可以看出, 随 着 $\mathrm{rGO}$ 添加量的增大，压痕尺寸先减小后逐渐增大， 在 $2 \mathrm{wt} \% \mathrm{rGO} / \mathrm{HAp}$ 样品中, 压痕对角线平均尺寸达

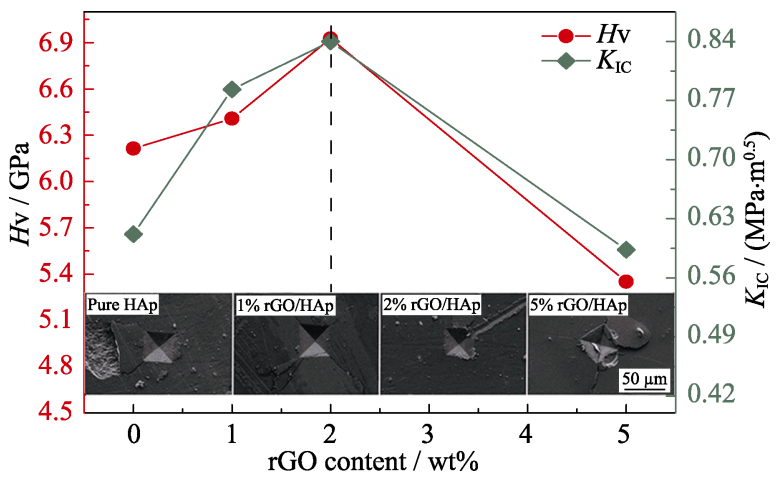

图 $7 \mathrm{rGO} / \mathrm{HAp}$ 复相生物陶瓷维氏硬度与断裂韧性变化曲线 Fig. 7 Vickers hardness and fracture toughness of rGO/HAp composite bioceramics

到最小值, 为 $54.38 \mu \mathrm{m}$, 且几乎看不到裂纹扩展, 表现出较好的力学性能。从图中可以看出, 随 $\mathrm{rGO}$ 含量的增多, 样品的硬度及断裂韧性均呈现出先升 高, 后急剧降低的变化趋势。其中, 添加 $2 \mathrm{wt} \% \mathrm{rGO}$ 的复相陶瓷表现出最佳的力学性能, 硬度与断裂韧 性分别达到 $6.97 \mathrm{GPa}$ 和 $0.84 \mathrm{MPa} \cdot \mathrm{m}^{1 / 2}$, 较纯相 $\mathrm{HAp}$ 陶瓷提高了 $11.5 \%$ 和 $37.3 \%$ 。因此，利用 $\mathrm{rGO}$ 增㓞 增强 HAp 生物陶瓷是完全可行的，结合图 4(a)的分 析可以推断, rGO 的拔出效应 ${ }^{[25]}$ 是导致复合材料综 合力学性能提高的主要原因。

\section{3 结论}

本研究通过等离子活化烧结成功制备了 $\mathrm{rGO} /$ HAp 复相生物陶瓷, 系统研究了 $\mathrm{rGO}$ 的添加对 HAp 基体物相结构、生物活性和力学性能的影响。得出 以下结论:

1) rGO 具有良好的生物相容性，对 HAp 优异的 生物活性几乎没有影响。 
2) rGO 可以有效改善 HAp 陶瓷的高温结构稳定 性，抑制 HAp 的分解与相转变。

3) 添加 $2 \mathrm{wt} \% \mathrm{rGO}$ 的复相陶瓷表现出最佳的 力学性能, 硬度与断裂韧性分别达到 $6.97 \mathrm{GPa}$ 和 $0.84 \mathrm{Mpa} \cdot \mathrm{m}^{1 / 2}$, 较纯相 HAp 陶瓷提高了 $11.5 \%$ 和 $37.3 \%$ 。经研究 rGO 的拔出效应是导致 HAp 陶瓷综 合力学性能提高的主要原因。

\section{参考文献:}

[1] YU P, BAO R Y, SHI X J, et al. Self-assembled high-strength hydroxyapatite/graphene oxide/chitosan composite hydrogel for bone tissue engineering. Carbohydrate Polymers, 2017, 155: 507-515.

[2] 徐晓宙. 生物材料学. 北京: 科学出版社, 2006: 70-75, 159169.

[3] DOU J H, ZHANG C Y, CHEN C Z, et al. Effects of sintering temperature on the properties of alumina/hydroxyapatite composites. Journal of Sol-Gel Science and Technology, 2017, 84(1): 2327.

[4] HU Z, TONG G, LIN D, et al. Graphene-reinforced metal matrix nanocomposites-a review. Materials Science \& Technology, 2016, 32(9): 930-953.

[5] GAO C D, LIU T T, SHUAI C J, et al. Enhancement mechanisms of graphene in nano-58S bioactive glass scaffold: mechanical and biological performance. Scientific Reports, 2014, 4: 4712-1-10.

[6] GURUNATHAN S, KIM J H. Synthesis, toxicity, biocompatibility, and biomedical applications of graphene and graphene-related materials. International Journal of Nanomedicine, 2016, 11: 1927-1945.

[7] CRISAN L, CRISAN B, SORITAU O, et al. In vitro study of biocompatibility of a graphene composite with gold nanoparticles and hydroxyapatite on human osteoblasts. Journal of Applied Toxicology, 2015, 35(10): 1200-1210.

[8] LEE S K, KIM H, SHIM B S. Graphene: an emerging material for biological tissue engineering. Carbon Letters, 2013, 14(2): 63-75.

[9] ZHU K P, SUN J, YE S, et al. A novel hollow hydroxyapatite microspheres/chitosan composite drug carrier for controlled release. Journal of Inorganic Materials, 2016, 31(4): 434-442.

[10] LIN J, CHEN X Y, HUANG P. Graphene-based nanomaterials for bioimaging. Advanced Drug Delivery Reviews, 2016, 105: 242254.

[11] WANG M H, ZHONG H B, FAN Y C, et al. Spark plasma sintering of bioactive $\mathrm{Ca}_{2} \mathrm{MgSi}_{2} \mathrm{O}_{7}$ ceramics. Journal of Inorganic Materials, 2017, 32(8): 825-830.
[12] ERIKSSON M, LIU Y, HU J F, et al. Transparent hydroxyapatite ceramics with nanograin structure prepared by high pressure spark plasma sintering at the minimized sintering temperature. Journal of the European Ceramic Society, 2011, 31(9): 1533-1540.

[13] HAN Y H, KIM B N, YOSHIDA H, et al. Spark plasma sintered superplastic deformed transparent ultrafine hydroxyapatite nanoceramics. Advances in Applied Ceramics, 2016, 115(3): 174-184.

[14] CHAMPION E. Sintering of calcium phosphate bioceramics. Acta Biomaterialia, 2013, 9(4): 5855-5875.

[15] ZHOU X J, ZHANG J L, WU H X, et al. Reducing graphene oxide via hydroxylamine: a simple and efficient route to graphene. Journal of Physical Chemistry C, 2011, 115(24): 11957-11961.

[16] LIU Y, ZHANG B, ZHANG L F, et al. Effect of hydrothermal etching processes on morphology and bioactivity of hydroxyapatite. Journal of Synthetic Crystals, 2016, 45(2): 441-446.

[17] 宋江风. 羟基磷灰石陶瓷及其复合材料的烧结行为及力学性能 研究. 长沙: 中南大学硕士学位论文, 2012.

[18] ZHANG L, LIU W W, YUE C G, et al. A tough graphene nanosheet/hydroxyapatite composite with improved in vitro, biocompatibility. Carbon, 2013, 61(11): 105-115.

[19] BAJPAI I, KIM D Y, HAN Y H, et al. Directional property evaluation of spark plasma sintered GNPs-reinforced hydroyapatite composites. Materials Letters, 2015, 158: 62-65.

[20] LIU Y, SHEN Z J. Dehydroxylation of hydroxyapatite in dense bulk ceramics sintered by spark plasma sintering. Journal of the European Ceramic Society, 2012, 32(11): 2691-2696.

[21] BONG S, KIM Y R, KIM I, et al. Graphene supported electrocatalysts for methanol oxidation. Electrochemistry Communications, 2010, 12(1): 129-131.

[22] BUZNIK V M, KOZLOVA S G, GABUDA S P, et al. Structural changes in carbonated hydroxyapatite at high temperatures as probed by ${ }^{1} \mathrm{H}$ NMR and Raman spectroscopy. Doklady Chemistry, 2007, 413(1): 64-67.

[23] LOPES J H, MAGALHAE J A, GOUUEIA R F, et al. Hierarchical structures of $\beta-\mathrm{TCP} / 45 \mathrm{~S} 5$ bioglass hybrid scaffolds prepared by gelcasting. Journal of the Mechanical Behavior of Biomedical Materials, 2016, 62: 10-23.

[24] LIU H Y, XI P X, XIE G Q, et al. Simultaneous reduction and surface functionalization of graphene oxide for hydroxyapatite mineralization. Journal of Physical Chemistry C, 2012, 116(5): 33343341.

[25] ZHU J T, WONG H M, YEUNG K W K, et al. Spark plasma sintered hydroxyapatite/graphite nanosheet and hydroxyapatite/ multiwalled carbon nanotube composites: mechanical and in vitro cellular properties. Advanced Engineering Materials, 2011, 13(4): 336-341. 